\title{
Cosmic Ray Energy Spectra and Mass Composition at the Knee - Recent Results from KASCADE -
}

\author{
K.-H. Kampert ${ }^{\mathrm{a}}{ }^{*}$, T. Antoni $^{\mathrm{b}}$, W.D. Apel ${ }^{\mathrm{c}}$, F. Badea ${ }^{\mathrm{c}}$, K. Bekk ${ }^{\mathrm{c}}$, A. Bercuci ${ }^{\mathrm{d}}$, H. Blümer bc, \\ H. Bozdog ${ }^{\text {c }}$, I.M. Brancus ${ }^{\text {d }}$, C. Büttner b, A. Chilingarian ${ }^{\text {e, K. Daumiller b }}{ }^{\text {, P. Doll }}$, R. Engel ${ }^{\text {, }}$, \\ J. Engler ${ }^{\text {c }}$, F. Feßler ${ }^{\text {b }}$, H.J. Gils ${ }^{\text {c }}$, R. Glasstetter ${ }^{\text {a }}$, A. Haungs ${ }^{\text {c }}$, D. Heck ${ }^{\text {c }}$, J.R. Hörandel ${ }^{\text {b }}$, \\ H.O. Klages ${ }^{\text {c }}$, G. Maier ${ }^{\text {c }}$, H.J. Mathes ${ }^{c}$, H.J. Mayer ${ }^{c}$, J. Milke ${ }^{\text {c }}$, M. Müller ${ }^{\text {c }}$, R. Obenland ${ }^{\text {c }}$, \\ J. Oehlschläger ${ }^{c}$, S. Ostapchenko ${ }^{b}$, M. Petcu ${ }^{\text {d }, ~ S . ~ P l e w n i a ~}{ }^{c}$, H. Rebel ${ }^{c}$, A. Risse ${ }^{f}$, M. Risse ${ }^{c}$, \\ M. Roth b, G. Schatz ${ }^{\text {c }}$, H. Schieler ${ }^{\text {c }}$, J. Scholz ${ }^{\text {c }}$, M. Stümpert ${ }^{\text {b }}$, T. Thouw ${ }^{\text {c }}$, H. Ulrich ${ }^{c}$, \\ J. van Buren ${ }^{c}$, A. Vardanyan ${ }^{\mathrm{e}}$, A. Weindl ${ }^{\mathrm{c}}$, J. Wochele ${ }^{\mathrm{c}}$, J. Zabierowski ${ }^{\mathrm{f}}$, S. Zagromski ${ }^{\mathrm{c}}$ \\ a Fachbereich C - Physik, Bergische Universität Wuppertal, 42097 Wuppertal, Germany \\ b Institut für Experimentelle Kernphysik, Universität Karlsruhe, 76021 Karlsruhe, Germany \\ c Institut für Kernphysik, Forschungszentrum Karlsruhe, 76021 Karlsruhe, Germany \\ d National Institute of Physics and Nuclear Engineering, 7690 Bucharest, Romania \\ e Cosmic Ray Division, Yerevan Physics Institute, Yerevan 36, Armenia \\ f Soltan Institute for Nuclear Studies, 90950 Lodz, Poland
}

Recent results from the KASCADE experiment on measurements of cosmic rays in the energy range of the knee are presented. Emphasis is placed on energy spectra of individual mass groups as obtained from an twodimensional unfolding applied to the reconstructed electron and truncated muon numbers of each individual EAS. The data show a knee-like structure in the energy spectra of light primaries (p, He, C) and an increasing dominance of heavy ones $(A \gtrsim 20)$ towards higher energies. This basic result is robust against uncertainties of the applied interaction models QGSJET and SIBYLL which are used in the shower simulations to analyse the data. Slight differences observed between experimental data and EAS simulations provide important clues for further improvements of the interaction models. The data are complemented by new limits on global anisotropies in the arrival directions of CRs and by upper limits on point sources. Astrophysical implications for discriminating models of maximum acceleration energy vs galactic diffusion/drift models of the knee are discussed based on this data.

\section{Introduction}

A puzzling and most prominent feature of the cosmic ray (CR) spectrum is the so-called knee, where the spectral index of the all-particle powerlaw spectrum changes from approximately -2.7 to -3.1 . Several models have been proposed in order to explain this feature shown in Fig.1 but none of them has managed to become broadly accepted. Some models focus on a possible change in the acceleration mechanism at the knee [123],

*email: kampert@uni-wuppertal.de e.g. due to the limiting energy defined by the size and magnetic field strength of the acceleration region $\left(E_{\max } \lesssim Z \times(B \times L)\right)$. Other ones discuss an increased leakage of CRs from the Galaxy due to a change in the confinement efficiency by galactic magnetic fields, e.g. 4567. Again, this results in a rigidity scaling of the knee according to the maximum confinement energy. Finally, a third group of models attributes the effect of the knee to CR interactions at their sources, during their propagation in the Galaxy, or in the upper atmosphere. Such scenarios include nuclear 


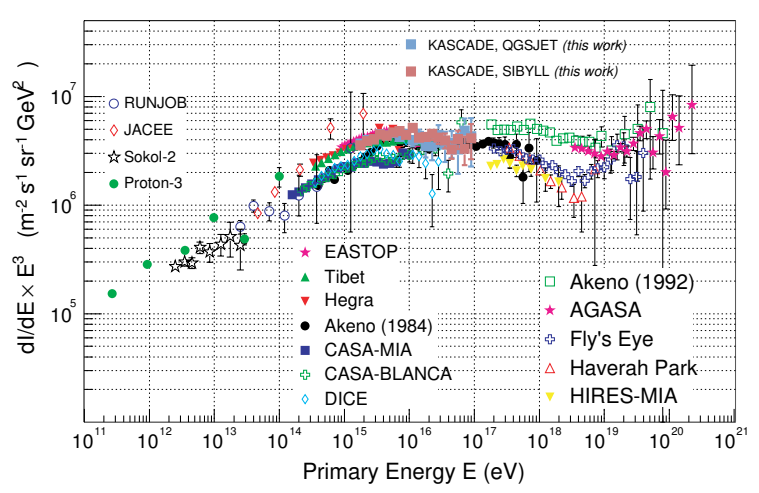

Figure 1. Compilation of the all-particle cosmic ray spectrum showing the knee, the suggested second knee, and the ankle of the CR spectrum (compilation by H. Ulrich).

photo-disintegration processes by UV-photons at the sources [8], interactions of CRs in dense fields of massive relic neutrinos 9, production of gravitons in high-energy pp collisions 10, etc. A recent review about this topic can be found e.g. in Refs. [1112].

To distinguish between these models and allowing to answer the long pressing question about the origin of cosmic rays and about the knee in their spectrum, high quality and high statistics data are required over an energy interval ranging from at least 0.5 to $500 \mathrm{PeV}$. It appears worthwhile to mention that solving the old problem about the origin of $\mathrm{CRs}$ in the $\mathrm{PeV}$ region is a prerequisite also for an understanding of the highest energies in the GZK-region. Due to the low flux involved at energies $\gtrsim 10^{14} \mathrm{eV}$, only extensive air shower (EAS) experiments are able to provide such data. In EAS experiments, primary CRs are only indirectly observed via their secondaries generated in the atmosphere. The most important experimental observables at ground are then the electromagnetic (electrons and photons), muonic, and hadronic components. In addition or alternatively, some experiments also detect photons originating from Cherenkov and/or fluorescence radiation of charged particles in the atmosphere. For a brief review about EAS observables and their experimental techniques the reader is referred to

\section{Refs. 131412 .}

Unfortunately, progress on interpreting EAS data has been modest mostly because of two reasons: Firstly, the EAS development is driven both by the poorly known high-energy hadronic interactions and their particle production in the very forward kinematical region as well as by uncertainties in the low energy interaction models influencing mostly the lateral particle density distribution functions [15]. Secondly, due to the stochastic nature of particle interactions, most importantly the height of the very first interaction in the atmosphere, EAS are subject to large fluctuations in particle numbers at ground. To make things even more complicated, the amount of fluctuations depends, amongst others, sensitively on the primary CR energy and mass [13]. Here, it is very important to realize that EAS fluctuations are not to be mistaken as random Gaussian errors associated with the statistics in the number of particles observed at ground. The latter one can be improved by the sampling area of an EAS experiment, while the former one is intrinsic to the EAS itself, carrying - for a sample of events - important information about the nature of the primary particle. Clearly, both kinds of fluctuations have to be accounted for in the data analysis of steeply falling energy spectra in order to avoid misinterpretations of the observations.

\section{Results from the KASCADE Experi- ment}

KASCADE (arlsruhe $\underline{\text { Shower }}$ Core and $\underline{\text { Array }}$ Detector) is a sophisticated EAS experiment for detailed investigations of primary CRs in the energy range of the knee. For reconstructing the CR energy and mass and for investigating highenergy hadronic interactions, KASCADE follows the concept of a multi-detector set-up providing as much complementary information as possible as well as redundancy for consistency tests. Most relevant for the results presented in this paper is the scintillator array comprising 252 detector stations of electron and muon counters arranged on a grid of $200 \times 200 \mathrm{~m}^{2}$. In total, it provides about $500 \mathrm{~m}^{2}$ of $e / \gamma$ - and $620 \mathrm{~m}^{2}$ of $\mu$-detector coverage. The detection thresholds for vertical incidence are 
$E_{e}^{\text {thr }} \simeq 5 \mathrm{MeV}$ and $E_{\mu}^{\text {thr }} \simeq 230 \mathrm{MeV}$. More details about the $e / \gamma$ - and $\mu$-detectors and all other detector components can be found in Ref. [16].

\subsection{Chemical Composition and Energy Spectra}

The traditional and perhaps most sensitive technique to infer the CR composition from EAS data is based on measurements of the electron $\left(N_{e}\right)$ and muon numbers $\left(N_{\mu}\right)$ at ground. It is well known [13] that for given energy, primary Fenuclei result in more muons and fewer electrons at ground as compared to proton primaries. Specifically, in the energy range and at the atmospheric depth of KASCADE, a Fe-primary yields about $30 \%$ more muons and almost a factor of two fewer electrons as compared to a proton primary. The basic quantitative procedure of KASCADE for obtaining the energy and mass of the cosmic rays is a technique of unfolding the observed twodimensional electron vs truncated muon number spectrum of Fig. 2 into the energy spectra of primary mass groups [17. The problem can be considered a system of coupled Fredholm integral equations of the form

$$
\begin{gathered}
\frac{d J}{d \lg N_{e} d \lg N_{\mu}^{\operatorname{tr}}}=\sum_{A} \int_{-\infty}^{+\infty} \frac{d J_{A}}{d \lg E} . \\
\cdot p_{A}\left(\lg N_{e}, \lg N_{\mu}^{\operatorname{tr}} \mid \lg E\right) \cdot d \lg E
\end{gathered}
$$

where the probability $p_{A}$

$$
\begin{aligned}
& p_{A}\left(\lg N_{e}, \lg N_{\mu}^{\operatorname{tr}} \mid \lg E\right)= \\
& \quad \int_{-\infty}^{+\infty} k_{A}\left(\lg N_{e}^{t}, \lg N_{\mu}^{\operatorname{tr}, \mathrm{t}}\right) d \lg N_{e}^{t} d \lg N_{\mu}^{\mathrm{tr}, \mathrm{t}}
\end{aligned}
$$

is another integral equation with the kernel function $k_{A}=r_{A} \cdot \epsilon_{A} \cdot s_{A}$ factorizing into three parts. Here, $r_{A}$ describes the shower fluctuations, i.e. the 2-dim distribution of electron and truncated muon number for fixed primary energy and mass, $\epsilon_{A}$ describes the trigger efficiency of the experiment, and $s_{A}$ the reconstruction probabilities, i.e. the distribution of $N_{e}$ and $N_{\mu}^{\mathrm{tr}}$ that is reconstructed for given true numbers $N_{e}^{t}, N_{\mu}^{\mathrm{tr}, \mathrm{t}}$ of electron and truncated muon numbers. The probabilities $p_{A}$ are obtained by parameterizations of EAS

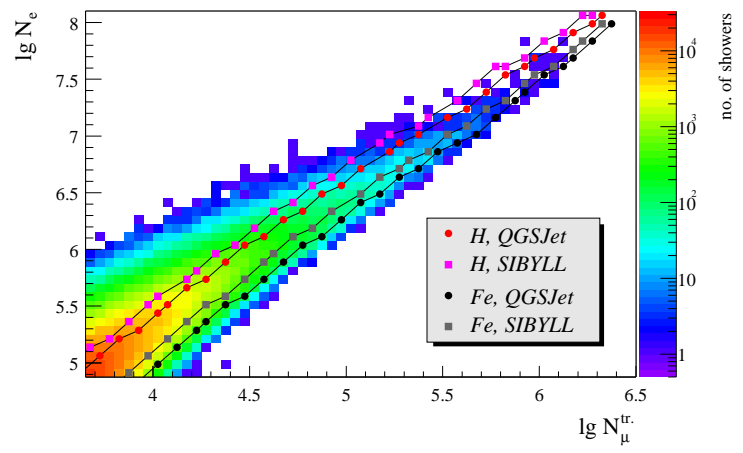

Figure 2. Two dimensional electron $\left(N_{e}\right)$ and truncated muon number $\left(N_{\mu}^{\mathrm{tr}}=\int_{40 \mathrm{~m}}^{200 \mathrm{~m}} \rho_{\mu}(r) d r\right)$ spectrum measured by the KASCADE array. Lines display the most probable values expected for proton and iron primaries according to CORSIKA simulations employing two different hadronic interaction models [17.

Monte Carlo simulations for fixed energies using a moderate thinning procedure as well as smaller samples of fully simulated showers for the input of the detector simulations. Because of the shower fluctuations mentioned above, unfolding of all 26 energy spectra ranging from protons to Fe-nuclei is clearly impossible. Therefore, 5 elements (p, $\mathrm{He}, \mathrm{C}, \mathrm{Si}, \mathrm{Fe}$ ) were chosen as representatives for the entire distribution. More mass groups do not improve the $\chi^{2}$-uncertainties of the unfolding but may result in mutual systematic biases of the reconstructed spectra 17.

The unfolding procedure is tested by using random initial spectra generated by Monte Carlo simulations. It has been shown [17 that knee positions and slopes of the initial spectra are well reproduced and that the discrimination between the five primary mass groups is sufficient. For scrutinizing the unfolding procedure, different mathematical ways of unfolding (Gold-algorithm, Bayes analyses, principle of maximum entropy, etc.) have been compared and the results are consistent 17. For generating the kernel functions a large number of EAS has been simulated [17/18 employing CORSIKA [19] with the hadronic in- 


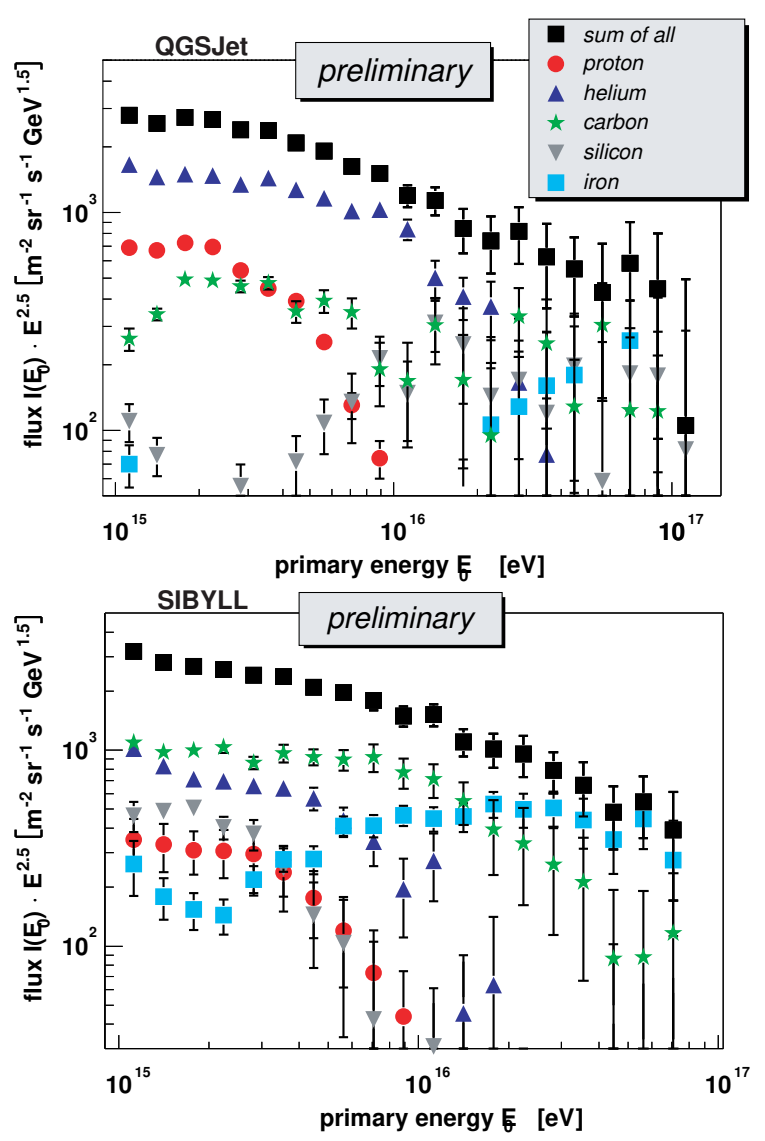

Figure 3. Results of the unfolding procedure using QGSJET (left) and SIBYLL (right) as hadronic interaction model [17.

teraction models QGSJET (version 2001) 20] and SIBYLL 2.1 21].

The result of the unfolding is presented in Fig. 3 for each of the two interaction models. Clearly, there are common features but also differences in the energy distributions obtained with the two interaction models. The all-particle spectra, also shown in Fig. 1] coincide very nicely and in both cases the knee is caused by the decreasing flux of the light primaries, corroborating results of an independent analysis of Ref. [22]. Tests using different data sets, different unfolding methods, etc. show the same behavior 18. As the most striking difference, SIBYLL suggests a more promi-

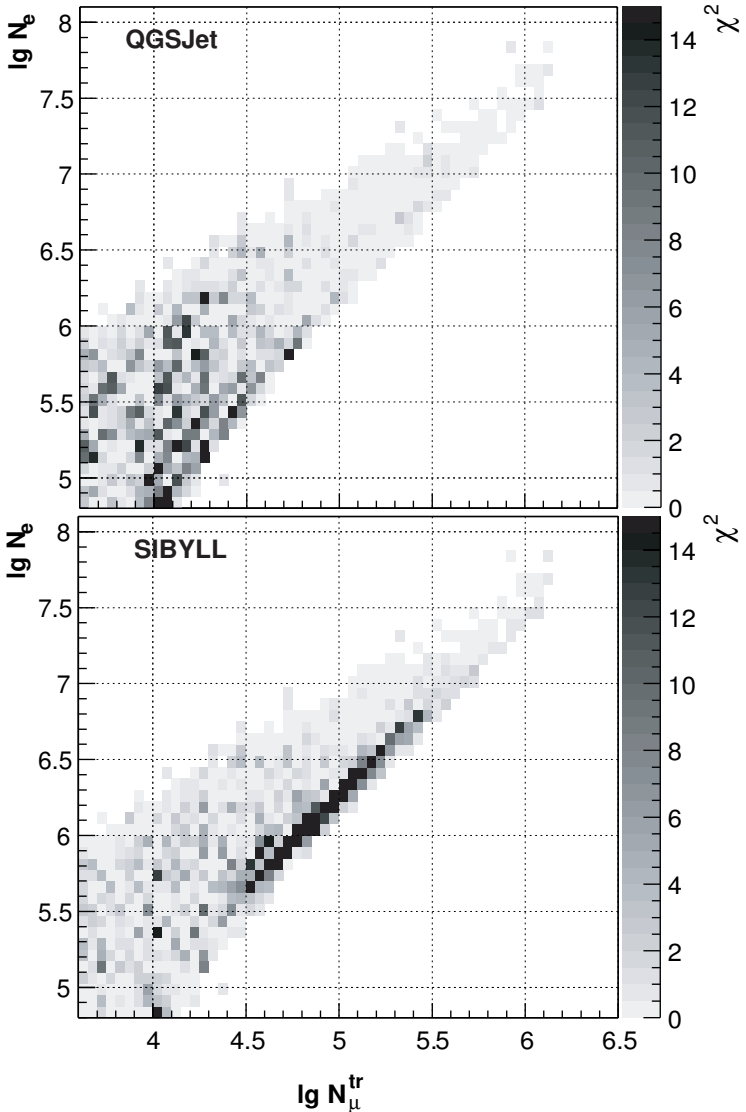

Figure 4. $\chi^{2}$ deviation between the forward folded reconstructed and measured $\left(N_{e}-N_{\mu}^{\mathrm{tr}}\right)$ data cells for the QGSJET (left) and SIBYLL (right) hadronic interaction models [17.

nent contribution of heavy primaries at high energies. This difference results from the different $N_{e}-N_{\mu}^{\mathrm{tr}}$ correlation shown in Fig.2 i.e. SIBYLL predicts higher electron and lower muon numbers for given primaries as compared to QGSJET.

Is there a way to judge which of the two models describes the data better? This is done most easily by comparing the residuals of the unfolded two-dimensional $N_{e}$ vs $N_{\mu}^{\mathrm{tr}}$ distributions with the actual data used as input to the unfolding (Fig.2). The result of such an analysis is presented in Fig. 4 in terms of $\chi^{2}$. The deviations reveal some deficiencies of QGSJET at low electron 
and muon numbers and they clearly demonstrate that SIBYLL encounters problems in describing the high- $N_{e}$ - low- $N_{\mu}^{\mathrm{tr}}$ tail of the experimental data at $10 \mathrm{PeV}$ and above [17. If not being prepared to accept an additional significant contribution of superheavy primaries $(A>60)$ required in case of SIBYLL simulations to fill the gap at high muon numbers, the results suggest a muon deficit (a/o electron excess) in this model. Definitely, this problem needs further attention and will be very important also for composition studies at higher energies 23.

With this caveats kept in mind, the KASCADE data favor an astrophysical interpretation of the knee and are in agreement with a constant rigidity of the knee position for the different primaries. Similar results were very recently obtained for an analysis of two-mass groups based on combined EAS-TOP / MACRO measurements [24, and were again confirmed for three mass groups from EAS-TOP electron and muon measurements [25]. Within the given error bars, the mean logarithmic masses of both experiments agree well with one another.

\subsection{Search for Anisotropies and Point Sources}

Additional information about the origin of CRs and their propagation in the galactic environment can be obtained from global anisotropies in their arrival directions. Model calculations show that diffusion of CRs in the galactic magnetic field can result in anisotropies on a scale of $10^{-4}$ to $10^{-2}$ depending on the energy of the particle, the strength and structure of the galactic magnetic field $[\underline{6}$, and on the source distribution. Since the diffusion scales again with the rigidity, a factor of 5-10 larger anisotropies are expected for protons as compared to iron primaries. This rigidity dependent diffusion is one of the possible explanations of the knee.

Because of the small anisotropy expected, a large data sample and careful data selection is necessary. About $10^{8}$ EAS events in the energy range from 0.7 to $6 \mathrm{PeV}$ were selected and studied in terms of Rayleigh amplitudes $A$ and phases $\Phi$ of the first harmonic. Neither for the full set of data nor for electron-rich and -poor EAS signifi-

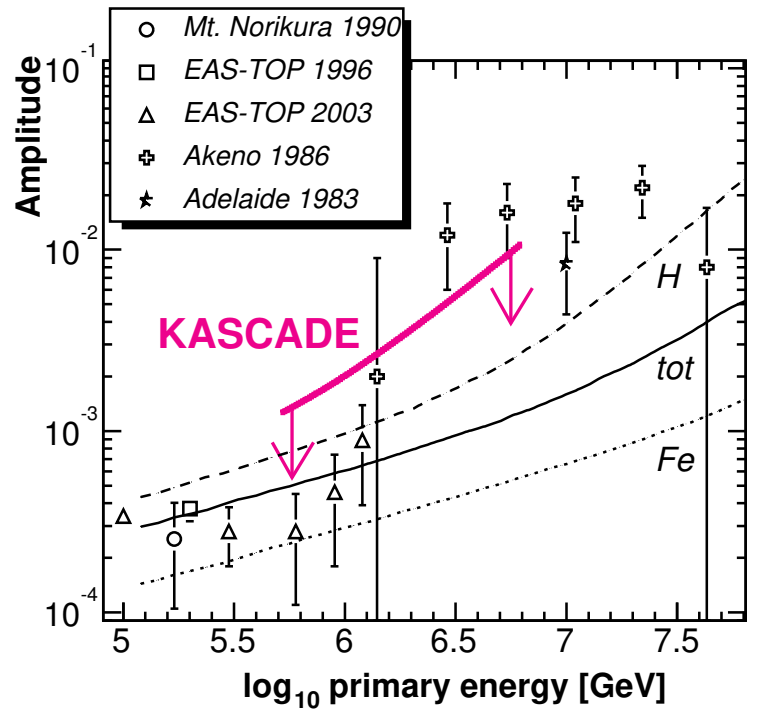

Figure 5. KASCADE upper limits (95\%) of Rayleigh amplitudes $A$ vs primary energy (bold line) compared to results from other experiments and to expectations from galactic $\mathrm{CR}$ diffusion (thin lines) [26.

cant Rayleigh amplitudes were found. The upper limit on the large scale anisotropy is depicted in Fig. [5 26 and is in line with results reported from other experiments. We shall come back to this result in the next section.

Even though the location of CR sources should be obscured due to the deflection of charged particles in the magnetic field of our galaxy, there is interest to perform point source searches. For example, neutrons are not deflected and can reach the Earth if their energy and hence decay length is comparable with the distance of the source. A decay length of $1 \mathrm{kpc}$ corresponds to a neutron energy of about $10^{17} \mathrm{eV}$. Also, by applying appropriate cuts to electron and muon numbers from EAS, searches for $\gamma$-ray point sources can be performed in the $\mathrm{PeV}$ range.

Such a study has been performed based on 47 Mio EAS with primary energies above $\sim 300$ $\mathrm{TeV}$. A certain region in the sky is then analyzed by comparing the number of events from the assumed direction with an expected number 


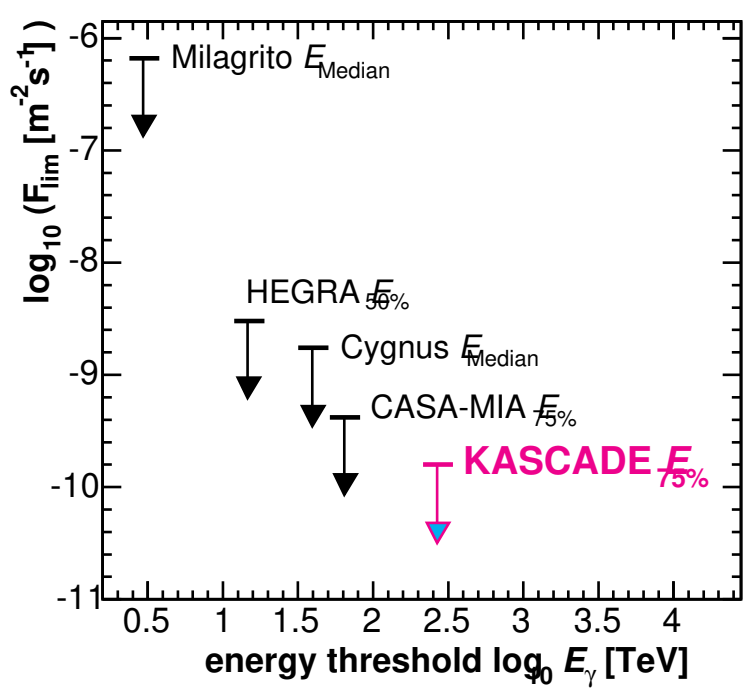

Figure 6. $90 \%$ upper limit for a point source moving through the zenith in comparison with results from other experiments [27]. Note the different definitions of the energy thresholds.

of background events. For the latter, the so-called time-shuffling method has been used. As a result, again no significant excess is found in the region of the galactic plane or for selected point source candidates. Assuming equal power laws in the energy spectra of background and source events, upper flux limits can be calculated for given energy thresholds. For a steady point source that transits the zenith, we obtain an upper flux limit of $3 \cdot 10^{-10} \mathrm{~m}^{-2} \mathrm{~s}^{-1}$ (see Fig. [6] [27. This is roughly 1-2 orders of magnitude larger than the Crab flux extrapolated to this energy.

Very recently, Chilingarian et al. reported the detection of a source of high-energy CRs in the Monogem ring [28]. Changing slightly our cuts in zenith angle to widen the declination range thereby covering the position of the source candidate, we find 742 events within an opening angle of $0.5^{\circ}$ around the suggested location with an expected number of 716 background events yielding an upper flux limit of $3 \cdot 10^{-10} \mathrm{~m}^{-2} \mathrm{~s}^{-1}$. Similar values are found when searching for an excess from the direction of the pulsar PSR B0656+14 29] located near the centre of the Monogem SNR.

\subsection{Implications for understanding the CR origin}

The new high quality data presented in the previous sections have revitalized the interest to understand both the origin of CRs in the knee region and the phenomenon of the knee structure itself.

This is because discriminating models of maximum acceleration energy from galactic diffusion/drift models of the knee or from particle physics interpretations require detailed inspection of knee structures seen in individual mass groups combined with precise measurements of $\mathrm{CR}$ anisotropies. Of particular interest are the energies of the spectral breaks, the power-law indices below and above the knee, and the smoothness of the turn-over regions. Even though, these goals are not yet achieved totally, important steps have been made towards it. Previous investigations were limited to inclusive CR all-particle spectra and to global changes of the mean logarithmic mass, $\ln A$, with primary energy. Such measurements appeared to be too insensitive for a convincing discrimination of models. Furthermore, in most cases not full distributions but only mean values of experimental and simulated distributions were compared to each other. A prominent example are plots of the shower maximum $X_{\max }$ vs primary energy. Obviously, deficiencies of hadronic interaction models remain unrecognized in such plots, unless EAS data are below proton or above iron simulations.

Very good examples demonstrating the discrimination power of the new data presented here and showing the amount of information contained in it is given by recent studies of Wick et al. 30 or Dar 31. Based on the earlier suggested connection between Gamma-Ray Bursts (GRBs) and ultrahigh-energy CRs 3233 they propose a model for the origin of CRs from $\sim 10^{14} \mathrm{eV} /$ nucleon up to the highest energies $\left(\gtrsim 10^{20} \mathrm{eV}\right)$. In that model, GRBs are assumed to inject $\mathrm{CR}$ protons and ions into the interstellar medium of star-forming galaxies - including the Milky Way - with a power-law spectrum extending to a maximum energy $\sim 10^{20} \mathrm{eV}$. High-energy CRs injected in the Milky Way diffuse and escape from our Galaxy. Ultra high-energy CRs with energies $\gtrsim 10^{17}$ to $10^{18} \mathrm{eV}$ that have Larmor radii 


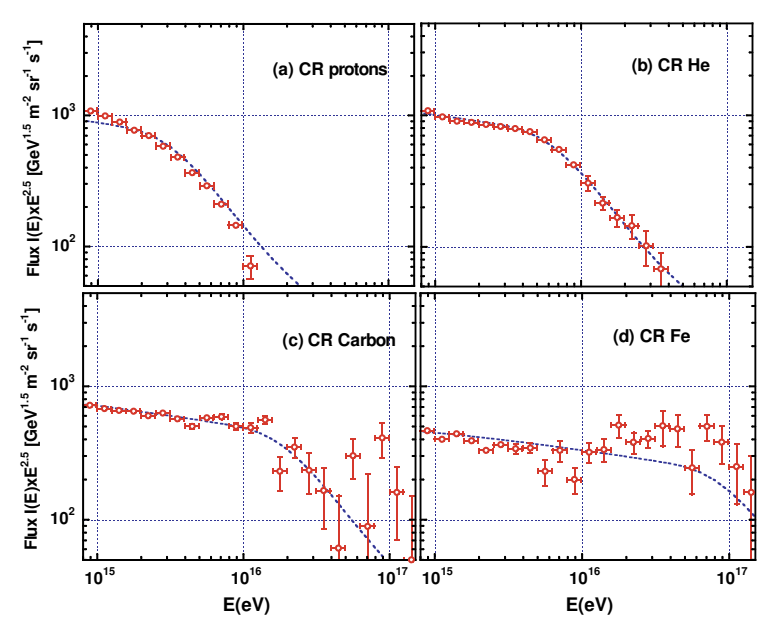

Figure 7. Fit of the GRB-model of Wick et al. 30 to the preliminary KASCADE data presented at ICRC 2001 34 35. In the model, a GRB occurred 210000 years ago at a distance of $500 \mathrm{pc}$ and injected $10^{52}$ ergs into CRs. The CRs isotropically diffuse with an energy-dependent mean-free-path in a MHD turbulence field.

comparable to the size scale of the galactic halo escape directly from the Milky Way and propagate almost rectilinearly through extragalactic space. By the same token, UHECRs produced from other galaxies can enter the Milky Way to be detected. UHECRs formed in GRBs throughout the universe then travel over cosmological distances and have their spectrum modified by energy losses, so an observer in the Milky Way will measure a superposition of UHECRs from extragalactic GRBs and HECRs produced in our Galaxy.

Thereby, the CR spectrum near the knee is understood by CRs trapped in the Galactic halo that were accelerated and injected by an earlier Galactic GRB. Assuming magnetohydrodynamical turbulence superposed to the galactic magnetic field, a fit of the model of Ref. 30 to the preliminary KASCADE data, shown in Fig. 7 suggests a 500 pc distant GRB that released $10^{52}$ ergs in CRs if the GRB took place about 210000 yrs ago. Keeping in mind the still large uncertainties of the data and some freedom of parameters in the model, there is remarkable accordance observed. In this model, the rigidity dependence of knee position is caused by galactic modulation effects. The GRB-model of Dar 31, on the other hand, predicts the knee to be proportional to the mass $A$ of $\mathrm{CR}$ primaries. This is because of the relativistic beaming effect (cannon balls) in the SN-jets of that model. It also fits the data within the present uncertainties with differences showing up mostly for protons and Helium $(Z / A=1,0.5$, respectively).

Since in these models very few or just a single galactic GRB is responsible for most of the CRs in the knee region, anisotropies in the arrival directions are expected on different levels, depending on the distance and age of the GRB. For example, the authors of Ref. [30] state that if an anisotropy below $\sim 0.2 \%$ is confirmed, then a number of implications follow. Either we are located near a rather recent GRB, which could be unlikely, or the CR energy release from GRBs is larger than the one given above 30. Thus, improving the anisotropy limits of the previous section would help to further pin down this model. Similarly, recent calculations of standard SN acceleration models show that global anisotropies well in excess even of $0.2 \%$ are expected for more realistic source distributions in the Galaxy, dependent on the structure of the magnetic fields [36]. The upper limits provided by the present data rule out already a large parameter space in these calculations.

However, before starting to over-interpret the data, particularly the energy spectra of mass groups, we should emphasize again their sensitivity to the applied interaction models. More work is still needed to improve the models and to arrive at smaller systematic uncertainties. On the other hand, the very valuable study of such models of CR origin demonstrates the informational content reached by present data.

\section{Summary and Outlook}

KASCADE has provided a wealth of new high quality EAS data in the knee region giving important insight into the origin of the knee and of CRs in general. Conclusive evidence has been 
reached on the knee being caused by light primaries $(p+\mathrm{He})$ mostly. Furthermore, the data are in agreement with a rigidity scaling of the knee position giving support to an astrophysical origin by either maximum acceleration or diffusion/drift models of propagation. Astrophysical parameters start to be constrained by the preliminary KASCADE data, as was demonstrated at the example of the GRB models of Refs. 30 31. Further important constrains result from measurements of large scale anisotropies of CRs and by new limits on point sources. Claims that were made in Ref. 28 about an excess of CRs from the Monogem SNR cannot be confirmed.

A particle physics interpretation of the knee appears to be excluded with a high level of confidence. For example, interactions of CRs with background particles or photo-disintegration in the Galaxy would produce an abundance of secondary protons and result in a light mass composition above the knee energy, a result which is in contradiction to the present data. Furthermore, $\overline{\nu_{e}}$ 's with a mass of $\simeq 0.5 \mathrm{eV} / c^{2}$ as are needed in the model of Ref. 9] appear to be excluded by recent WMAP and $2 \mathrm{dF}$ data for neutrinos in case of degenerated masses, with the latter being suggested by recent oscillation and possibly by a neutrinoless double-beta experiment (for a recent discussion of this topic the reader is referred to Refs. 37, 38).

Presently, more data and more observables are being analyzed within KASCADE, particularly in terms of composition analyses employing reconstructions of the muon production height [39]. Together with measurements of energetic hadrons in the central calorimeter, the unfolding technique of electron and muon numbers in EAS has become a powerful tool to reconstruct the properties of primary particles in EAS and it also provides important clues on how to improve the hadronic interaction models employed in CORSIKA air shower simulations.

KASCADE-Grande has just started its routinely data taking and will extend the measurements up to $10^{18} \mathrm{eV}$, thereby allowing to verify the existence of the putative Iron knee marking the so-called second knee in the all-particle CR spectrum [041]. This, together with improved statistics for anisotropy measurements will allow to confront astro- and particle-physics motivated models of the knee in much more detail to the experimental data as has been possible up to now.

Furthermore, the use of radio antennas complementing the experimental KASCADE-Grande set-up may open a new window to future EAS observations on large scales 42 41. Interesting first observations have been made and are presently being analyzed in detail.

Acknowledgement: It is a pleasure to thank the organizers of the meeting for financial support and for their invitation to participate in a very interesting and fruitful meeting conducted in a pleasant atmosphere. This work is supported by Forschungszentrum Karlsruhe, the German Ministry for Research and Education (Grant 05 CU1VK1/9), and by a Polish KBN grant for the years 2004-6.

\section{REFERENCES}

1. P.O. Lagage and C.J. Cesarsky, Astron. Astrophys. 118 (1983) 223, Astron. Astrophys. 125 (1983) 249.

2. P.L. Biermann, Inv. Rap. High. Papers, $23^{\text {rd }}$ ICRC Calgary (1993) 45.

3. L.C. Drury, Astron. Astrophys. 287 (1994) 959.

4. J. Wdowczyk and A.W. Wolfendale, J. Phys. G10 (1984) 1453.

5. V.S. Ptuskin et al., Astron. Astrophys. 268 (1993) 1453.

6. J. Candia, E. Roulet, and L.N. Epele, J. High Energy Phys. 12 (2002) 33.

7. E. Roulet, Int. J. Mod. Phys. A19 (2004) 1133 (2003).

8. J. Candia, L.N. Epele, and E. Roulet, $A s-$ tropart. Phys. 17 (2002) 23.

9. R. Wigmans, Astropart. Phys. 19 (2003) 379.

10. D. Kazanas and A. Nikolaidis, Gen. Rel. Grav. 35 (2001) 1117.

11. K.-H. Kampert, Acta Phys. Hung. New Ser. Heavy Ion Phys. 14 (2001) 203

12. A. Haungs, H. Rebel, and M. Roth, Rep. Prog. Phys. 66 (2003) 1145.

13. K.-H. Kampert, J. Phys. G27 (2001) 1663.

14. S. Swordy et al., Astropart. Phys. 18 (2002) 
129.

15. H.-J. Drescher, M. Bleicher, S. Soff, H. Stöcker, Astropart. Phys. 21 (2004) 87.

16. T. Antoni et al., (KASCADE Coll.), Nucl. Instr. Meth. A513 (2003) 490.

17. H. Ulrich, Doctoral Thesis at University Karlsruhe, 2003; H. Ulrich et al., (KASCADE Coll.), Nucl. Phys. B (Proc. Suppl.) 122 (2003) 218.

18. M. Roth, H. Ulrich et al., (KASCADE-Coll.), $28^{\text {th }}$ ICRC (Tsukuba) 1, (2003), 131.

19. D. Heck et al., Report FZKA 6019, (1998)

20. N.N. Kalmykov, S.S. Ostapchenko, Yad.Fiz. 56, (1993), 105.

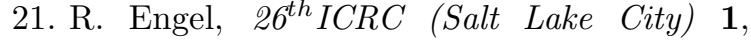
(1999), 415.

22. T. Antoni et al., (KASCADE Coll.), Astrop. Phys. 16 (2002) 373.

23. A.A. Watson, talk presented at this meeting; astro-ph/0408110

24. M. Aglietta et al., (EAS-TOP \& MACRO Coll.), Astrop. Phys. 20 (2004) 641

25. G. Navarra, priv. comm. (2004)

26. G. Maier, Doctoral Thesis at University Karlsruhe, 2003; T. Antoni et al., (KASCADE Coll.), Astrophys. J. 604 (2004) 687; astro-ph/0312375

27. G. Maier, Doctoral Thesis at University Karlsruhe, 2003; T. Antoni et al., (KASCADE Coll.), Astrophys. J. 608 (2004) 865

28. A. Chilingarian, H. Martirosian, G. Gharagyozyan, ApJ 597 (2003) L291

29. S.E. Thorsett et al., ApJ 592 (2003) L71

30. S.D. Wick, C.D. Dermer, A. Atoyan, Astropart. Phys. 21 (2004) 125

31. Arnon Dar, astro-ph/0408310 and private communication.

32. M. Vietri, ApJ 453 (1995) 883

33. E. Waxman, Phys. Rev. Lett. 75 (1995) 386

34. K.-H. Kampert et al., (KASCADE-Coll.), Invited, Rapporteur, and Highlight papers of ICRC 2001, p240.

35. H. Ulrich et al., (KASCADE-Coll.), $27^{\text {th }}$ ICRC, Hamburg, (2001) 97.

36. V.S. Ptuskin et al., 28 $8^{\text {th }}$ ICRC (Tsukuba), (2003), 1933.

37. S. Hannestad, New J. of Phys. 6 (2004) 108

38. G.L. Fogli et al., astro-ph/0408045
39. C. Büttner et al., (KASCADE-Coll.), $28^{\text {th }}$ ICRC (Tsukuba), (2003), 33.

40. K.-H. Kampert et al., (KASCADE-Grande Coll.), Nucl. Phys. B (Proc. Suppl.) 122 (2003) 422.

41. F. Badea et al., (KASCADE-Grande Coll.), talk presented at this meeting.

42. A. Horneffer et al., 28 $8^{\text {th }}$ ICRC (Tsukuba), (2003), 969. 